\title{
Identification and analysis of unitary loss of long- established protein-coding genes in Poaceae shows evidences for biased gene loss and putatively functional transcription of relics
}

\author{
Yi Zhao ${ }^{1}$, Liang Tang ${ }^{1,2}$, Zhe Li $i^{3}$, Jinpu Jin ${ }^{1}$, Jingchu Luo ${ }^{1}$ and Ge Gao ${ }^{1 *}$
}

\begin{abstract}
Background: Long-established protein-coding genes may lose their coding potential during evolution ("unitary gene loss"). Members of the Poaceae family are a major food source and represent an ideal model clade for plant evolution research. However, the global pattern of unitary gene loss in Poaceae genomes as well as the evolutionary fate of lost genes are still less-investigated and remain largely elusive.

Results: Using a locally developed pipeline, we identified 129 unitary gene loss events for long-established protein-coding genes from four representative species of Poaceae, i.e. brachypodium, rice, sorghum and maize. Functional annotation suggested that the lost genes in all or most of Poaceae species are enriched for genes involved in development and response to endogenous stimulus. We also found that 44 mutated genomic loci of lost genes, which we referred as relics, were still actively transcribed, and of which $84 \%$ (37 of 44) showed significantly differential expression across different tissues. More interestingly, we found that there were totally five expressed relics may function as competitive endogenous RNA in brachypodium, rice and sorghum genome.

Conclusions: Based on comparative genomics and transcriptome data, we firstly compiled a comprehensive catalogue of unitary gene loss events in Poaceae species and characterized a statistically significant functional preference for these lost genes as well showed the potential of relics functioning as competitive endogenous RNAs in Poaceae genomes.
\end{abstract}

Keywords: Unitary gene loss, Poaceae, Competitive endogenous RNA

\section{Background}

Both point mutations and large deletions can disrupt open reading frame of long-established protein-coding genes in a species or a clade, resulting in the loss of coding potential and genetic functions, which is referred to as "unitary gene loss" [1-4]. Although it is taken as "deleterious" by conventional wisdom, several studies demonstrated that unitary gene loss may also contribute to evolutionary novelty and even be adaptive, e.g. immune responses [1] and pathogen interactions $[5,6]$ in humans, the origin of a partially reproductively isolated race in Drosophila melanogaster [7,8], and the

\footnotetext{
*Correspondence: gaog@mail.cbi.pku.edu.cn

${ }^{1}$ State Key Laboratory of Protein and Plant Gene Research, College of Life Science, Center for Bioinformatics, Peking University, Beijing 100871, People's Republic of China

Full list of author information is available at the end of the article
}

generation of self-fertilisation in Arabidopsis [9]. Several systematic profiles for unitary gene loss have been done in human and rodents [1-4]. Interestingly, Marques et al. found more than half of lost genes in rodents retained expression and played a new role as competitive endogenous RNAs (ceRNAs) to regulate the expression of other transcripts by altering microRNA availability [4], which suggests a possible functional mechanism for these relics.

Members of the Poaceae are a major food source, and this family represents an ideal model clade for plant evolutionary analysis [10]. Recently, two genome-wide studies investigated loss of duplicated gene copies in plants $[11,12]$. However, the global picture of unitary loss for long-established protein-coding genes in Poaceae remain largely unknown and elusive. 
In this study, we first developed a novel comparative genomics-oriented pipeline to identify unitary loss of longestablished gene in the four representative Poaceae, i.e. brachypodium, rice, sorghum and maize. Taking Arabidopsis, poplar and grape as out groups, we focused on lineagespecific loss of long-established protein-coding genes which have been conserved for more than 160 million years ago (Mya) [13] and only got lost after divergence of Poaceae clade. Employing stringent criteria, we identified 129 unitary gene loss events (UGLEs) in the four genomes, with 47, 27, 23 and 32 UGLEs identified in brachypodium, rice, sorghum and maize genome, respectively. Expression profiling analysis showed that the relics of 44 lost genes were still actively transcribed, 37 of which showed significantly varied expression pattern across different tissues. After removing these more likely due to partially degenerated promoters, we still identified 30 reliable expressed relics. Among them, we identified one brachypodium relic, two rice relics and two sorghum relics as putative ceRNAs, suggesting that unitary gene loss may contribute to the origin of functional non-coding RNAs.

\section{Results}

\section{Identification of unitary gene loss events}

Unitary loss of long-established protein-coding genes can be identified by both orthologous mapping of protein sequences $[1,3]$, and syntenic mapping of gene loci $[2,4]$. To date, most studies take pseudogenes, i.e. relics, as proxies for identifying gene loss events [14]. However, if the gene loss occurred long ago or evolved very rapidly, it would be difficult to identify relics based on homologous searching alone. Moreover, if a gene is lost through intra-chromosomal recombination [15], it is also hard to detect relics based on left homologous fragments. In addition, syntenic mapping cannot identify lost genes without significant synteny, inducing falsenegative cases caused by genome rearrangement such as insertion, deletion and translocation event [16].

To address these challenges, we developed a novel pipeline independent of relics and synteny to identify unitary gene loss events in Poaceae. Using this pipeline, we firstly identified candidate unitary gene loss events based on orthologous relationships among different species. Three out groups including Arabidopsis, poplar and grape were used and the principle of parsimony $[17,18]$ was adopted to distinguish gene loss events from gene gains in other lineages (Figure 1a, also see Methods for more details).

Beginning with 45,589 orthologous groups containing orthologous genes in four Poaceae species and three out-group species, we identified 742 candidate unitary gene loss events. In the case of possible false-positives caused by transposable elements or mitochondrial and chloroplast-related genes, we further removed 21 cases, in which 7, 2, 4 and 8 from brachypodium, rice, sorghum and maize respectively, and generated a dataset containing 721 candidate unitary gene loss events (see Methods for more details).

To further filter out false-positive unitary gene loss events due to incorrectly annotated orthologous relationships, we ran PseudoPipe [19] using the entire genome sequence of the species as the object and protein sequence of an orthologous counterpart of the lost gene from the adjacent species (e.g., brachypodium for rice) as the query. We then extracted genomic hits for 424 candidate unitary gene loss events. Of these events, 248 candidates with genic hits were removed, because they may primarily occur due to incomplete genomic annotation rather than bona fide unitary gene loss. For the rest 176 candidates that matched intergenic regions, we removed an additional 115 events that had intact open reading frame (ORF) matches. The remaining 61 disabler-harbouring candidates, which contains at least one ORF-disrupting site, such as a frameshift or premature stop codon, were classified as relic-retaining unitary gene loss events. Furthermore, the remaining 297 candidate unitary gene loss events without genomic hits between the genome and orthologous counterparts are taken to analysis for validation. Only candidates which can be located in the genome based on synteny mapping across brachypodium, rice, sorghum and maize are considered as relic-lacking unitary gene loss events. After excluding candidates in synteny-inconsistent and gapped regions, we obtained 68 relic-lacking unitary gene loss events.

Finally, we identified 129 unitary gene loss events, including 61 relic-retaining and 68 relic-lacking ones (Figure 1b). Most unitary gene loss events occurred recently and only in one species, except for the unitary gene loss in orthologous group 11453 occurred before the divergence of brachypodium and rice and unitary gene loss in orthologous group 8968 occurred before the divergence of sorghum and maize. The entire pipeline is in Figure 1c while available at the website http://gene-loss.cbi.pku.edu.cn, and information on the identified unitary gene loss events is included in Additional file 1, providing a resource for the gene loss community in plants.

\section{Functions of lost genes in wild and cultivated species are different}

The biological functions of lost genes can be inferred by their orthologous counterparts [3]. Based on ortholog mapping, we functionally annotated 124 out of 129 lost genes using Plant GO slim terms of their Arabidopsis orthologs retrieved from AmiGO (see Additional file 2). Subsequently, we took GO slim distribution among genes in each Poaceae species as the background and conducted GO slim enrichment analysis through Fisher's 


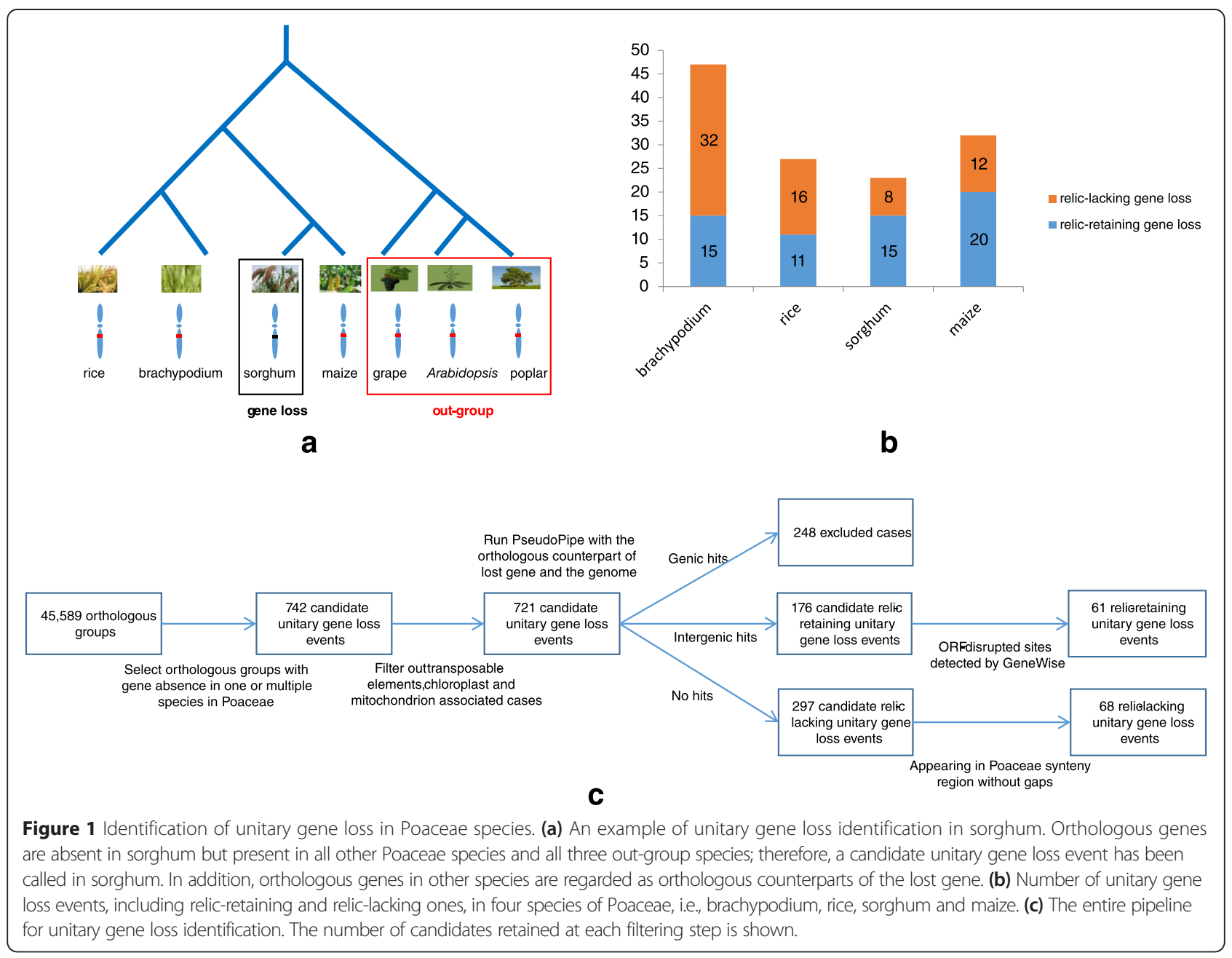

Exact test and FDR multiple test correction, to see whether there are any functional preferences of lost genes (see Methods for more details, Table 1).

First of all, lost functions in all or most of species are commonly enriched with development, such as multicellular organismal development (GO:0007275, FDR-corrected $\mathrm{p}$-value $=0.000017$ in brachypodium, FDR-corrected $\mathrm{p}$ value $=0.00095$ in rice, FDR-corrected $p$-value $=0.013$ in sorghum and FDR-corrected $\mathrm{p}$-value $=0.0070$ in maize) and post-embryonic development (GO:0009791, FDR-corrected $\mathrm{p}$-value $=0.00013$ in brachypodium, FDR-corrected $\mathrm{p}$-value $=0.012$ in sorghum and FDRcorrected p-value $=0.00065$ in maize). Detailed inspection showed that most of lost genes annotated as multicellular organismal development and post-embryonic development were associated with reproductive process, such as vegetative to reproductive phase transition of meristem (GO:0010228), embryo development ending in seed dormancy (GO:0009793), seed dormancy process (GO:0010162) and seed germination (GO:0009845). In addition to development, lost functions in most of species are also commonly enriched with response to endogenous stimulus (GO:0009719, FDR-corrected p-value $=0.0037$ in brachypodium, FDR-corrected $\mathrm{p}$-value $=0.0049$ in rice and FDR-corrected p-value $=0.0060$ in maize). Further inspection showed most of this kind of lost genes involved in response to varieties of hormones (i.e. auxin, gibberellin, cytokinin, abscisic acid, ethylene, jasmonic acid and brassinosteroid) and chitin.

On the other hand, we also found several speciesspecific functional enrichment for lost genes. The function of sequence-specific DNA binding transcription factor activity (GO:0003700, FDR-corrected p-value $=0.0076)$ is enriched in lost genes of brachypodium uniquely. By checking the annotation of Arabidopsis thaliana orthologs of this kind of lost genes in plant Transcription Factor Database (PlantTFDB) [20], we found that lost genes encoded transcription factors which are important for development of plant, such as ones in WOX and MYB families. Another species-specific enriched function of lost 
Table 1 Plant GO slim enrichment of lost genes in Poaceae species

\begin{tabular}{|c|c|c|c|c|c|c|}
\hline Class & Plant GO slim annotation & Species & $\begin{array}{l}\text { Observed lost } \\
\text { number }\end{array}$ & $\begin{array}{l}\text { Expected lost } \\
\text { number }\end{array}$ & $\begin{array}{l}\text { Observed/ } \\
\text { Expected }\end{array}$ & $\begin{array}{l}\text { FDR-corrected } \\
\text { pvalue }\end{array}$ \\
\hline \multirow{8}{*}{$\begin{array}{l}\text { Shared by four } \\
\text { species }\end{array}$} & multicellular organismal development & brachypodium & 13 & 2.9 & 4.6 & 0.0000 \\
\hline & & rice & 6 & 0.9 & 6.7 & 0.0009 \\
\hline & & sorghum & 5 & 1.1 & 4.4 & 0.0133 \\
\hline & & maize & 6 & 1.4 & 4.4 & 0.0070 \\
\hline & cellular component organization & brachypodium & 13 & 2.9 & 4.5 & 0.0000 \\
\hline & & rice & 6 & 1.8 & 3.4 & 0.0135 \\
\hline & & sorghum & 5 & 1.1 & 4.7 & 0.0126 \\
\hline & & maize & 11 & 1.7 & 6.3 & 0.0000 \\
\hline \multirow{6}{*}{$\begin{array}{l}\text { Shared by three } \\
\text { species }\end{array}$} & post-embryonic development & brachypodium & 9 & 1.6 & 5.5 & 0.0001 \\
\hline & & sorghum & 4 & 0.7 & 6.1 & 0.0126 \\
\hline & & maize & 6 & 0.8 & 7.7 & 0.0006 \\
\hline & response to endogenous stimulus & brachypodium & 7 & 1.7 & 4.2 & 0.0037 \\
\hline & & rice & 4 & 0.6 & 7.1 & 0.0049 \\
\hline & & maize & 5 & 0.9 & 5.8 & 0.0060 \\
\hline \multirow{4}{*}{$\begin{array}{l}\text { Shared by two } \\
\text { species }\end{array}$} & anatomical structure morphogenesis & brachypodium & 12 & 1.1 & 11.2 & 0.0000 \\
\hline & & rice & 4 & 0.3 & 12.9 & 0.0009 \\
\hline & cell cycle & brachypodium & 5 & 0.4 & 11.3 & 0.0003 \\
\hline & & sorghum & 4 & 0.2 & 21.5 & 0.0004 \\
\hline \multirow[t]{10}{*}{ Species-specific } & biosynthetic process & brachypodium & 15 & 8.3 & 1.8 & 0.0300 \\
\hline & cell differentiation & brachypodium & 8 & 0.6 & 12.4 & 0.0000 \\
\hline & cell growth & brachypodium & 4 & 0.6 & 6.7 & 0.0076 \\
\hline & embryo development & brachypodium & 5 & 0.6 & 8.6 & 0.0010 \\
\hline & flower development & brachypodium & 8 & 0.6 & 13.6 & 0.0000 \\
\hline & growth & brachypodium & 7 & 0.7 & 9.6 & 0.0000 \\
\hline & response to external stimulus & brachypodium & 7 & 1.7 & 4.0 & 0.0045 \\
\hline & $\begin{array}{l}\text { sequence-specific DNA binding } \\
\text { transcription factor activity }\end{array}$ & brachypodium & 7 & 1.9 & 3.6 & 0.0076 \\
\hline & carbohydrate metabolic process & rice & 5 & 0.9 & 5.8 & 0.0035 \\
\hline & transport & maize & 8 & 3.3 & 2.4 & 0.0412 \\
\hline
\end{tabular}

genes in brachypodium is response to external stimulus (GO:0009605, FDR-corrected p-value $=0.0045)$. Most of this kind of genes involved in response to environmental stress and phytopathogen, such as anthocyanin accumulation in tissues in response to UV light (GO:0043481), cellular process regulating host cell cycle in response to virus(GO:0060154), defense response to bacterium (GO:0042742) and response to other organism (GO:0051707). Interestingly, genes involved in the carbohydrate metabolic process (GO:0005975) prefer to be lost only in rice genome (FDR-corrected p-value $=0.0035)$, while transport genes (GO:0006810) are enriched in lost genes of maize $($ FDR-corrected $\mathrm{p}$-value $=0.0412)$.

\section{Several relics are still under active transcription}

Although relics remaining after unitary gene loss have lost their protein-coding potential and thus cannot function as proteins, studies demonstrated that they may still being transcribed and play other roles in the species at the transcript level, such as non-coding RNAs [21]. To explore this phenomenon in Poaceae, we detected expression signatures for 61 identified relics.

We used four RNA-seq datasets with the same samples in four Poaceae species, including leaves, emerging inflorescence (pre-pollination tassel and pre-emergence cob for maize), early inflorescence (post-pollination tassel and post-emergence cob for maize), anther, pistil 
(mature silk and ovule for maize), seeds harvested 5 days after pollination (DAP), seeds harvested 10 DAP, embryo harvested 25 DAP and endosperm harvested 25 DAP to identify expressed relics [22,23]. We found there were 12 out of 15 relics in brachypodium, 9 out of 11 relics in rice, 13 out of 15 relics in sorghum and 10 out of 20 relics in maize expressed respectively. Compared with their orthologous counterparts, these expressed relics possess similar expression level and tissue specificity (Figure 2; see Additional file 3). Interestingly, we also found 11 out of 12 expressed relics in brachypodium, 9 out of 9 expressed relics in rice, 12 out of 13 expressed relics in sorghum and 5 out of 10 expressed relics in maize expressed with significant variation among different samples.

The transcription of recently evolved relics may be spurious due to their partially degenerated promoter regions $[24,25]$. To test this possibility, we first directly compared the expression pattern between the relic and its orthologous counterpart in the neighbouring species. After excluding four brachypodium relics, one sorghum relic and one maize relic, whose orthologous counterparts have no expression signatures detected in these four data sets, we found the expression of only seven relics (one for brachypodium, two for rice and four for maize) showed significant similar pattern with that of their orthologous counterpart (two-tailed Student's $t$ test, Pearson $\mathrm{R}>0.6$, FDR-corrected $\mathrm{p}$-value $<0.05$ ) (Figure 3; see Additional file 4). Besides comparison the expression pattern between relic and its orthologous counterpart, we also checked the conservation of promoter region of expressed relics. We identified there was one brachypodium relic possessing significantly conserved promoter region than protein-coding genes through Wilcoxon Rank Sum test (GERP score, FDRcorrected p-value < 0.05) (Figure 3; see Additional file 4). These eight relics with similar expression pattern or conserved promoters were removed from further analysis.

It has been reported that transcripts losing coding potential could function as ceRNAs in rodents $[4,26]$. We found there were one brachypodium relic, two rice relics and two sorghum relics encode different microRNA response elements and could be a plausible target for corresponding microRNAs, implying that they might become ceRNAs and thereby function as microRNA decoys $[27,28]$. Consistent with the hypothesis $[4,26,29]$, we found there were proteincoding genes harbouring the same microRNA response elements in three prime untranslated region and expressing with positive correlation for each of these relics (Table 2). Further check showed that there were no out-paralog relationships between these relics and protein-coding genes, thus the sharing of the same microRNA response element is not due to the homology between them, further suggesting the possible ceRNA roles for these transcribed relics.

\section{Discussion}

Based on orthologous relationships in Poaceae with outgroup species in monocotyledons and the application of filtering criteria, we identified a total of 129 UGLEs of longestablished protein-coding gene in four Poaceae genomes, including 61 relic-retaining ones and 68 relic-lacking ones. The inclusion of out-group species in monocotyledons can ensure the long-established status of lost genes and make it
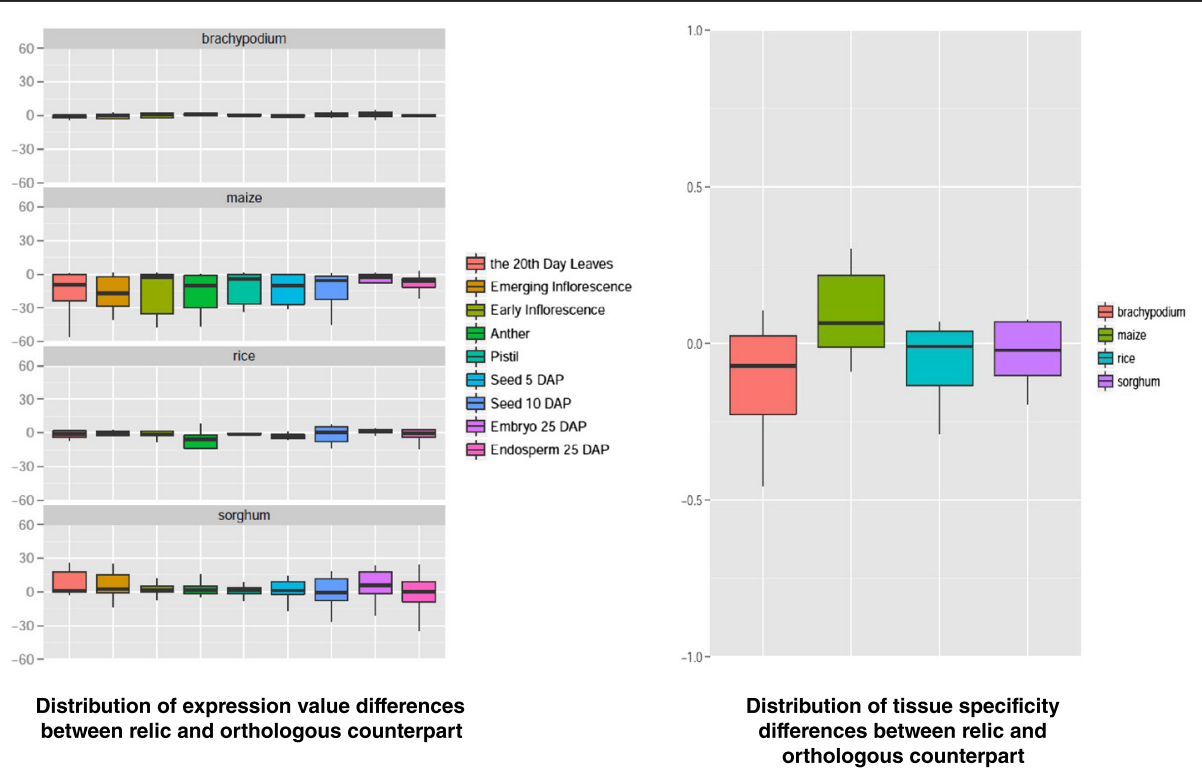

Figure 2 Distribution of differences associated with expression value and tissue specificity between relics and orthologous counterparts in Poaceae species. 


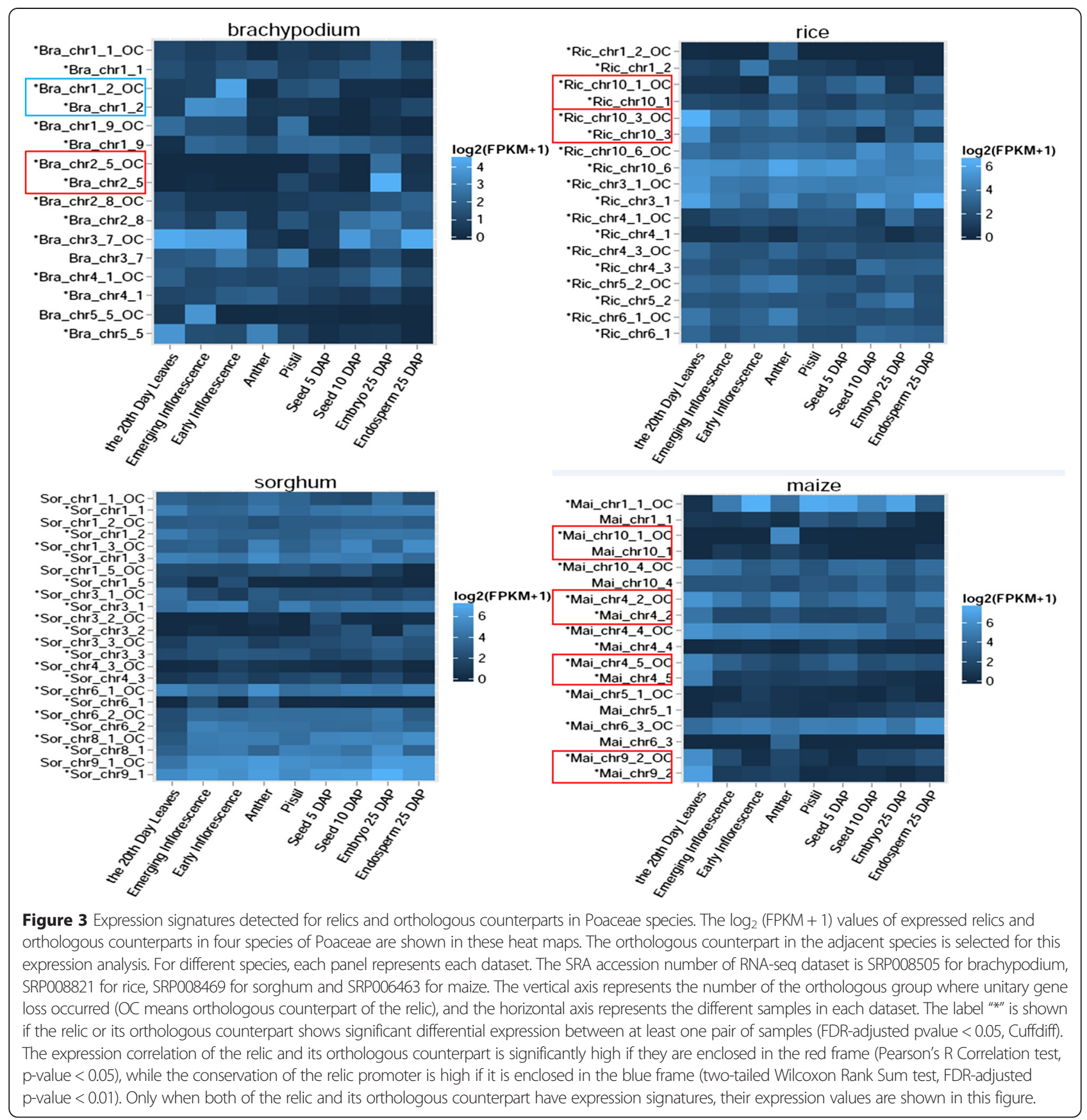

possible to identify unitary gene loss based on the principle of parsimony.

To minimize false positive rate, we adopted very strict criteria for detecting unitary gene loss in this study. For relic-retaining unitary gene loss, we filtered out those cases with evident genic matches or that lacked ORFdisrupting sites. For relic-lacking unitary gene loss, we filtered out those cases that could not be located based on synteny data. Of these unitary gene loss events, we found that 46, 26, 22 and 31 events specifically occurred in brachypodium, rice, sorghum and maize, respectively.
Considering that the divergence time of brachypodiumrice is 40 million years [30] and that of sorghum-maize is 12 million years [31], we estimated the gene death rate $\left(V_{D}\right)$ for each species after the latest divergence. We found that the average death rate was 1.6 genes per million years, with the lowest (0.7) rates observed in rice and the highest (2.6) rates occurring in maize (Figure 4). Consistent with previous observations [11], the $V_{D}$ for brachypodium (1.2) was more than $70 \%$ higher than that of rice, which may contribute to the relatively small genome size of brachypodium [30] and suggest on-going 
Table 2 List of relics as potential ceRNAs

\begin{tabular}{llllll}
\hline ID & miRNA & Orthologous group of relic & $\begin{array}{l}\text { Gene with the same microRNA } \\
\text { response element }\end{array}$ & Expression correlation & $\begin{array}{l}\text { FDR-corrected pvalue } \\
\text { for the correlation }\end{array}$ \\
\hline Bra_chr4_1 & bdi-miR5181a-3p & 7462 & BRADI2G55497 & 0.00 \\
Bra_chr4_1 & bdi-miR5181a-3p & 7462 & BRADI3G27520 & 0.89 & 0.01 \\
Bra_chr4_1 & bdi-miR5203 & 7462 & BRADI1G77247 & 0.85 & 0.04 \\
Bra_chr4_1 & bdi-miR5203 & 7462 & BRADI2G55497 & 0.59 & 0.00 \\
Ric_chr5_2 & osa-miR530-5p & 578 & LOC_Os01g16180 & 0.89 & 0.02 \\
Ric_chr1_2 & osa-miR531b & 395 & LOC_Os08g35210 & 0.02 \\
Sor_chr1_5 & sbi-miR5384 & 6806 & Sb01g018950 & 0.83 & 0.04 \\
Sor_chr1_1 & sbi-miR5568f-5p & 6026 & Sb08g015530 & 0.76 & 0.02 \\
\hline
\end{tabular}

gene deletion happened in brachypodium [11]. Moreover, except for brachypodium, the accelerated gene death rate is accompanied by large genome size, suggesting the potential mechanism that large genome is less stable than small one and possesses faster evolved gene content in Poaceae [32,33].

Based on Plant GO slim enrichment analysis, both common and species-specific functional preferences of lost genes were detected in Poaceae. Being consistent with previous observation in human genome [3], the functions of development associated with the reproductive process are enriched in all of these four Poaceae genomes. Interestingly, we noticed that Schnable et al. have speculated that a special case of gene loss (the reciprocal loss of homeologous genes) may contribute to the radiation of Poaceae lineage but failed to find supporting evidence [11], while Muir and Hahn have suggested the reciprocal gene loss contributed less to increased speciation rates [34]. Our observation on the enrichment of reproductive function in unitarily lost genes, on the other hand, may provide a new angle for the hypothesis.

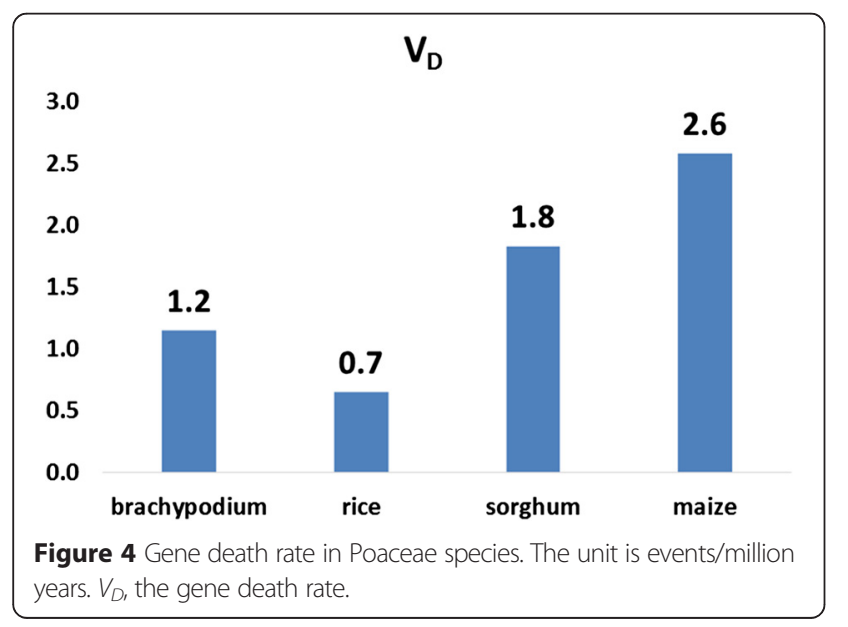

Besides the function of associated with reproductive process, the function associated with response to endogenous stimulus is also enriched in brachypodium, rice and maize. Commonly, most of lost genes annotated with this function are related with response to varieties of plant hormones, the key factor for plants to regulate signalling pathways involved in response to varieties of biotic and abiotic stresses [35]. Interestingly, we also found genes involved in response to endogenous chitin stimulus, a proxy for fungal infection [36,37], lost in brachypodium and maize genome, suggesting a potential role of gene loss in the adaption of changing environments stresses like biotic stresses.

There are also multiple species-specific functional preference of lost genes in different Poaceae species, especially in brachypodium. In brachypodium, genes with the molecular function as sequence-specific DNA binding transcription factor activity are preferred to lose. Detailed analysis showed that lost genes in this class are annotated as transcription factors essential for plant development based on their orthologous counterpart in Arabidopsis thaliana (see Additional file 5). It suggests that brachypodium reshapes its mode of development associated with reproductive process via adjustment the gene content associated with transcriptional regulation. Moreover, genes associated with response to external stimulus are also preferred to lose in brachypodium. It is consistent with the fact that brachypodium is a wild plant, which faces more rapid changing of environmental stress and phytopathogen. Therefore, similar with genes processing the function of response to endogenous stimulus, this kind of genes in brachypodium are also becoming the evolutionary hot spots.

It has been demonstrated that some relics retained after gene loss could continue to express and function as noncoding RNAs [21,38-42]. However, "functional expression" of relics as non-coding RNA must be distinguished from "leaky expression" of relics generated from partially degenerated promoter region. In Arabidopsis and rice, it has 
been demonstrated that recently lost duplicated genes could retain significantly lower expression $[24,25]$. However, in our data set, we found expressed relics possess similar expression level and expression width compared with their orthologous counterparts. Moreover, we also found 37 out of 44 expressed relics possess significantly varied expression values across different samples, implying their expression is under control of specific regulation. The similar expression level, expression width of relics compared with their orthologous counterparts and the specific regulation of expression suggest their expression is not likely just transcriptional noise [43].

On the other hand, the differential expression can also solely result from the left over regulatory sequence ("partially degenerated promoter"). To test the hypothesis, we further compared the expression pattern between relic and its orthologous counterpart across different samples, and assessed the evolutionary conservation of expressed relics' promoters directly. We found the expression of more than $65 \%$ (30 out of 44 ) relics cannot be fully explained by the partially degeneration promoter hypothesis, implying neofunctionalization of these relics $[44,45]$. Interestingly, within these 30 reliable expressed relics, we found there were one brachypodium relic, two rice relics and two sorghum relics may function as ceRNAs and undertake the role of microRNA decoys, suggesting a source of novel non-coding RNAs.

\section{Conclusions}

Based on orthologous relationships and several strict filtering criteria, we systematically identified the unitary loss of long-established protein-coding genes in four representative species of Poaceae, i.e. brachypodium, rice, sorghum and maize, discovering 129 unitary gene loss events in total. By Plant GO slim enrichment analysis, we found genes with function of development associated with reproductive process, while response to endogenous stimulus are commonly preferred to lose in all or most of Poaceae species, suggesting unitary gene loss might affect the features of reproduction and stimulus response in different Poaceae species. In addition, after excluding expressed relics are more likely due to partially degenerated promoter region, we totally assured 30 expressed relics which may bring evolutionary novelty to the species. Furthermore, among these 30 expressed relics, we found one brachypodium relic, two rice relics and two sorghum relics may function as ceRNAs, indicating a potential contribution of unitary gene loss to the origin of functional non-coding RNAs. Systematic identification and analysis of the unitary loss of long-established protein-coding genes in these four species will elucidate the global perspective and unique features of this evolutionary process in Poaceae.

\section{Methods}

\section{Identification of unitary gene loss}

Preliminary unitary loss of long-established proteincoding gene was identified based on the orthologous relationships among four representative species in Poaceae, i.e., brachypodium, rice, sorghum and maize, and three species in out-group, i.e., Arabidopsis, poplar and grape. The orthologous groups are retrieved from Ensembl Plants release 15 [46] including genes annotated in Brachypodium distachyon v1.0, Oryza sativa Japonica MSU6, Sorghum bicolor v1.0, Zea mays AGPv2, Vitis vinifera IGGP_12x, Arabidopsis thaliana TAIR10 and Populus trichocarpa JGI2.0.

The absence of orthologous genes in a species may either represent gene loss in that species or gene gain in other species. However, based on the principle of parsimony, if orthologous genes are present in multiple species but absent in just one, a single gene loss event is more plausible than multiple simultaneous gene gain events $[17,18]$. This strategy needs more species to be considered. Therefore, we assigned preliminary unitary gene loss events based on the orthologous relationships between Poaceae species brachypodium, rice, sorghum and maize, and out-group species Arabidopsis, poplar and grape. Briefly, if a gene is absent in one Poaceae species but its orthologous present in all other Poaceae and out-group species, a preliminary UGLE will be assigned to the branch, and all functional genes in the same orthologous group will be taken as the orthologous counterparts of this lost gene. In case of false positive, preliminary UGLE was further filtered by removing relics with intact ORF identified by GeneWise [47], relics with genic hits of an orthologous counterpart from the adjacent species, as well as transposable, mitochondrial and chloroplast elements.

If there were no any genomic hits of orthologous counterparts on the genome for a preliminary lost gene, its genomic location was inferred based on the synteny among Poaceae species produced by Schnable et al. [11]. In case of artefacts due to incomplete genome sequence/annotation, we excluded all preliminary reliclacking UGLE with either gene annotation or genomic gaps in the synteny regions of neighbour genomes from further analysis.

\section{Plant GO slim annotation and analysis of the functional preferences of lost genes}

Because there were no direct functional annotations for the lost gene, the original function of lost gene was annotated by Plant GO slim of its orthologous counterparts in Arabidopsis. Then, GO slims of protein-coding genes in each Poaceae species were extracted as the background for statistics test. Through Fisher's Exact test and FDR multiple test correction, enriched GO slims were identified for lost genes in each Poaceae species. 


\section{Detection of expression signatures for relics}

Four RNA-seq datasets (i.e. SRP008505 for brachypodium, SRP008821 for rice, SRP008469 for sorghum and SRP006463 for maize) were retrieved from NCBI were used to detect expression signatures for relics. All RNAseq reads of each dataset were mapped to the genome using TopHat [48] and called differential expression by Cuffdiff [49]. Only relic with fragments per kilobase of sequence per million reads mapped (FPKM) value greater than one in at least one sample was considered as expressed relic. Wilcoxon Signed Rank test and FDR correction were conducted to determine whether there were significant differences of the expression level and tissue specificity (i.e. the maximum value of fractional expression of locus in one tissue relative to the sum of all tissues) between relic and its orthologous counterpart.

\section{Conservation analysis of the promoter region for relics}

The $2 \mathrm{~kb}$ intergenic region upstream of the 5 ' boundary of relic or protein-coding gene was taken as the putative promoter. GERP (Genomic Evolutionary Rate Profiling) conservation scores $[50,51]$ of the promoter region of relic were calculated based on multiple alignment of promoter region of relic and its orthologous counterparts, which is available at the website http://gene-loss.cbi.pku.edu.cn, and a species tree constructed via RAxML [52,53]. The tree was used as the input of GERP program to calculate the neutral rate by summing all of the lengths of the tree. To evaluate whether the conservation of the promoter region of relic was significantly different from that of protein-coding gene, GERP scores of the promoter region of protein-coding genes in the same species were also calculated and considered as the background of statistics test.

\section{Analysis of ceRNA features for relics}

The microRNA response element (MRE) on relic and 3' untranslated region (3' UTR) of protein-coding gene was predicted by TAPIR [54] with the default parameters. The microRNA sequences were retrieved from miRBase release 20 [55], while the 3' UTR sequences of proteincoding genes were retrieved from BioMart of Ensembl Plants release 15 [56]. Then, Pearson's R Correlation test and FDR multiple test correction were conducted to identify expressed relic possessing significantly positive expression correlation with protein-coding gene harbouring the same MRE on 3' UTR.

\section{Availability of supporting data}

The multiple alignment of promoter region of relic and its orthologous counterparts is available in the website http://gene-loss.cbi.pku.edu.cn/, while all other supporting data is available as additional files.

\section{Additional files}

Additional file 1: Detail List of unitary gene loss events in Poaceae clade.

Additional file 2: Plant GO slim and GO annotation for lost genes. Additional file 3: Wilcoxon Signed Rank test result for expression value and tissue specificity between relic and its orthologous counterpart.

Additional file 4: Expression correlation between the relic and its orthologous counterpart, as well as the GERP score of relic promoter. Additional file 5: Lost transcription factors in brachypodium.

\section{Abbreviations}

ceRNA: Competitive endogenous RNA; Mya: Million years ago; UGLE: Unitary gene loss event; ORF: Open reading frame; DAP: Days after pollination; $V_{D}$ : Gene death rate; FPKM: Fragments per kilobase of sequence per million reads mapped; GERP: Genomic Evolutionary Rate Profiling; MRE: microRNA response element; 3' UTR: 3' untranslated region; FDR: False discovery rate.

\section{Competing interests}

The authors declare that they have no competing interests.

\section{Authors' contributions}

YZ, LT and GG designed this study. YZ built the pipeline for unitary gene loss identification, performed the analyses of unitary gene loss events for the four representative species in Poaceae (i.e., brachypodium, rice, sorghum and maize), and drafted the manuscript. $L T, Z L, J J, J L$ and $G G$ participated in the revision of the manuscript. All authors read and approved the final manuscript.

\section{Acknowledgements}

We thank Manyuan Long at the University of Chicago for helpful discussion and comments. This work was supported by the China National 973 Program [2011CBA01103]; 863 Programs [2006AA02Z334]; and the State Key Laboratory of Protein and Plant Gene Research. The research of G.G. was supported in part by the National Program for Support of Top-notch Young Professionals. J.J. was supported partly by the China Postdoctoral Science Foundation Grant [2014M560017]. Liang Tang is partly supported by grant from the Chongqing Natural Science Foundation (cstc2012jjA80017).

\section{Author details}

${ }^{1}$ State Key Laboratory of Protein and Plant Gene Research, College of Life Science, Center for Bioinformatics, Peking University, Beijing 100871, People's Republic of China. ${ }^{2}$ Current address: College of Horticulture and Landscape Architecture, Southwest University, Chongqing 400715, People's Republic of China. ${ }^{3}$ State Key Laboratory of Systematic and Evolutionary Botany, Institute of Botany, Chinese Academy of Sciences, Beijing 100093, People's Republic of China.

Received: 21 January 2015 Accepted: 31 March 2015

Published online: 18 April 2015

\section{References}

1. Wang X, Grus WE, Zhang J. Gene losses during human origins. PLoS Biol. 2006;4(3):e52.

2. Zhu J, Sanborn JZ, Diekhans M, Lowe CB, Pringle TH, Haussler D. Comparative genomics search for losses of long-established genes on the human lineage. PLoS Comput Biol. 2007;3(12):e247.

3. Zhang ZD, Frankish A, Hunt T, Harrow J, Gerstein M. Identification and analysis of unitary pseudogenes: historic and contemporary gene losses in humans and other primates. Genome Biol. 2010;11(3):R26.

4. Marques AC, Tan J, Lee S, Kong L, Heger A, Ponting CP. Evidence for conserved post-transcriptional roles of unitary pseudogenes and for frequent bifunctionality of mRNAs. Genome Biol. 2012;13(11):R102.

5. Tournamille C, Colin Y, Cartron JP, Le Van KC. Disruption of a GATA motif in the Duffy gene promoter abolishes erythroid gene expression in Duffy-negative individuals. Nat Genet. 1995;10(2):224-8.

6. Dean M, Carrington M, Winkler C, Huttley GA, Smith MW, Allikmets R, et al. Genetic restriction of HIV-1 infection and progression to AIDS by a deletion allele of the CKR5 structural gene. Hemophilia Growth and Development 
Study, Multicenter AIDS Cohort Study, Multicenter Hemophilia Cohort Study, San Francisco City Cohort, ALIVE Study. Science. 1996;273(5283):1856-62.

7. Takahashi A, Tsaur SC, Coyne JA, Wu Cl. The nucleotide changes governing cuticular hydrocarbon variation and their evolution in Drosophila melanogaster. Proc Natl Acad Sci U S A. 2001;98(7):3920-5.

8. Greenberg AJ, Moran JR, Coyne JA, Wu Cl. Ecological adaptation during incipient speciation revealed by precise gene replacement. Science. 2003;302(5651):1754-7.

9. Shimizu KK, Cork JM, Caicedo AL, Mays CA, Moore RC, Olsen KM, et al. Darwinian selection on a selfing locus. Science. 2004;306(5704):2081-4.

10. Buell CR. Poaceae genomes: going from unattainable to becoming a model clade for comparative plant genomics. Plant Physiol. 2009;149(1):111-6.

11. Schnable JC, Freeling M, Lyons E. Genome-wide analysis of syntenic gene deletion in the grasses. Genome biol evol. 2012;4(3):265-77

12. De Smet R, Adams KL, Vandepoele K, Van Montagu MC, Maere S, Van de Peer Y. Convergent gene loss following gene and genome duplications creates single-copy families in flowering plants. Proc Natl Acad Sci U S A. 2013;110(8):2898-903.

13. Hedges SB, Dudley J, Kumar S. TimeTree: a public knowledge-base of divergence times among organisms. Bioinformatics. 2006;22(23):2971-2.

14. Schrider DR, Costello JC, Hahn MW. All human-specific gene losses are present in the genome as pseudogenes. J Comput Biol. 2009;16(10):1419-27.

15. Woodhouse MR, Schnable JC, Pedersen BS, Lyons E, Lisch D, Subramaniam $\mathrm{S}$, et al. Following tetraploidy in maize, a short deletion mechanism removed genes preferentially from one of the two homologs. PLoS Biol. 2010;8(6), e1000409.

16. Jung S, Jiwan D, Cho I, Lee T, Abbott A, Sosinski B, et al. Synteny of Prunus and other model plant species. BMC Genomics. 2009;10:76.

17. Mirkin BG, Fenner TI, Galperin MY, Koonin EV. Algorithms for computing parsimonious evolutionary scenarios for genome evolution, the last universal common ancestor and dominance of horizontal gene transfer in the evolution of prokaryotes. BMC Evol Biol. 2003;3:2

18. Boussau B, Karlberg EO, Frank AC, Legault BA, Andersson SG. Computational inference of scenarios for alpha-proteobacterial genome evolution. Proc Natl Acad Sci U S A. 2004;101(26):9722-7.

19. Zhang Z, Carriero N, Zheng D, Karro J, Harrison PM, Gerstein M. PseudoPipe: an automated pseudogene identification pipeline. Bioinformatics. 2006;22(12):1437-9.

20. Jin J, Zhang H, Kong L, Gao G, Luo J. PlantTFDB 3.0: a portal for the functional and evolutionary study of plant transcription factors. Nucleic Acids Res. 2014:42(Database issue):D1182-1187.

21. Zheng D, Gerstein MB. The ambiguous boundary between genes and pseudogenes: the dead rise up, or do they? Trends Genet. 2007;23(5):219-24.

22. Davidson RM, Hansey CN, Gowda M, Childs KL, Lin H, Vaillancourt B, et al. Utility of RNA Sequencing for Analysis of Maize Reproductive Transcriptomes. Plant Gen. 2011:4(3):191-203.

23. Davidson RM, Gowda M, Moghe G, Lin H, Vaillancourt B, Shiu SH, et al. Comparative transcriptomics of three Poaceae species reveals patterns of gene expression evolution. Plant J. 2012;71(3):492-502.

24. Thibaud-Nissen F, Ouyang S, Buell CR. Identification and characterization of pseudogenes in the rice gene complement. BMC Genomics. 2009;10:317

25. Zou C, Lehti-Shiu MD, Thibaud-Nissen F, Prakash T, Buell CR, Shiu SH. Evolutionary and expression signatures of pseudogenes in Arabidopsis and rice. Plant Physiol. 2009;151(1):3-15.

26. Long M, Zhang L. Why rodent pseudogenes refuse to retire. Genome Biol. 2012;13(11):178.

27. Ebert MS, Sharp PA. Emerging roles for natural microRNA sponges. Curr Biol. 2010;20(19):R858-861.

28. Marques AC, Tan J, Ponting CP. Wrangling for microRNAs provokes much crosstalk. Genome Biol. 2011;12(11):132.

29. Poliseno L, Salmena L, Zhang J, Carver B, Haveman WJ, Pandolfi PP. A coding-independent function of gene and pseudogene mRNAs regulates tumour biology. Nature. 2010;465(7301):1033-8.

30. International Brachypodium I. Genome sequencing and analysis of the model grass Brachypodium distachyon. Nature. 2010;463(7282):763-8.

31. Swigonova Z, Lai J, Ma J, Ramakrishna W, Llaca V, Bennetzen JL, et al. Close split of sorghum and maize genome progenitors. Genome Res. 2004;14(10A):1916-23
32. Luo MC, Deal KR, Akhunov ED, Akhunova AR, Anderson OD, Anderson JA, et al. Genome comparisons reveal a dominant mechanism of chromosome number reduction in grasses and accelerated genome evolution in Triticeae. Proc Natl Acad Sci U S A. 2009;106(37):15780-5.

33. Massa AN, Wanjugi H, Deal KR, O'Brien K, You FM, Maiti R, et al. Gene space dynamics during the evolution of Aegilops tauschii, Brachypodium distachyon, Oryza sativa, and Sorghum bicolor genomes. Mol Biol Evol. 2011;28(9):2537-47.

34. Muir CD, Hahn MW. The limited contribution of reciprocal gene loss to increased speciation rates following whole-genome duplication. Am Nat. 2015;185(1):70-86.

35. Bari R, Jones JD. Role of plant hormones in plant defence responses. Plant Mol Biol. 2009;69(4):473-88.

36. Wan J, Zhang XC, Stacey G. Chitin signaling and plant disease resistance. Plant Signal Behav. 2008:3(10):831-3.

37. Eckardt NA. Chitin signaling in plants: insights into the perception of fungal pathogens and rhizobacterial symbionts. Plant Cell. 2008;20(2):241-3.

38. Korneev SA, Park JH, O'Shea M. Neuronal expression of neural nitric oxide synthase (nNOS) protein is suppressed by an antisense RNA transcribed from an NOS pseudogene. J Neurosci. 1999;19(18):7711-20.

39. Korneev S, O'Shea M. Evolution of nitric oxide synthase regulatory genes by DNA inversion. Mol Biol Evol. 2002;19(8):1228-33.

40. Korneev SA, Straub V, Kemenes I, Korneeva El, Ott SR, Benjamin PR, et al. Timed and targeted differential regulation of nitric oxide synthase (NOS) and anti-NOS genes by reward conditioning leading to long-term memory formation. J Neurosci. 2005;25(5):1188-92.

41. Duret L, Chureau C, Samain S, Weissenbach J, Avner P. The Xist RNA gene evolved in eutherians by pseudogenization of a protein-coding gene. Science. 2006;312(5780):1653-5.

42. Chaumeil J, Waters PD, Koina E, Gilbert C, Robinson TJ, Graves JA. Evolution from XIST-independent to XIST-controlled X-chromosome inactivation: epigenetic modifications in distantly related mammals. PLoS One. 2011;6(4), e19040.

43. Hangauer MJ, Vaughn IW, McManus MT. Pervasive transcription of the human genome produces thousands of previously unidentified long intergenic noncoding RNAs. PLoS Genet. 2013;9(6), e1003569.

44. Duarte JM, Cui L, Wall PK, Zhang Q, Zhang X, Leebens-Mack J, et al. Expression pattern shifts following duplication indicative of subfunctionalization and neofunctionalization in regulatory genes of Arabidopsis. Mol Biol Evol. 2006;23(2):469-78.

45. Li Z, Zhang H, Ge S, Gu X, Gao G, Luo J. Expression pattern divergence of duplicated genes in rice. BMC Bioinf. 2009;10 Suppl 6:S8.

46. Kersey PJ, Staines DM, Lawson D, Kulesha E, Derwent P, Humphrey JC, et al. Ensembl Genomes: an integrative resource for genome-scale data from non-vertebrate species. Nucleic Acids Res. 2012;40(Database issue):D91-97.

47. Birney E, Clamp M, Durbin R. GeneWise and Genomewise. Genome Res. 2004;14(5):988-95.

48. Trapnell C, Pachter L, Salzberg SL. TopHat: discovering splice junctions with RNA-Seq. Bioinformatics. 2009;25(9):1105-11.

49. Trapnell C, Williams BA, Pertea G, Mortazavi A, Kwan G, van Baren MJ, et al. Transcript assembly and quantification by RNA-Seq reveals unannotated transcripts and isoform switching during cell differentiation. Nat Biotechnol. 2010;28(5):511-5.

50. Cooper GM, Stone EA, Asimenos G, Green ED, Batzoglou S, Sidow A Distribution and intensity of constraint in mammalian genomic sequence. Genome Res. 2005;15(7):901-13.

51. Davydov EV, Goode DL, Sirota M, Cooper GM, Sidow A, Batzoglou S. Identifying a high fraction of the human genome to be under selective constraint using GERP++. PLoS Comput Biol. 2010;6(12):e1001025.

52. Stamatakis A. RAxML-VI-HPC: maximum likelihood-based phylogenetic analyses with thousands of taxa and mixed models. Bioinformatics. 2006;22(21):2688-90.

53. Stamatakis A, Hoover $P$, Rougemont J. A rapid bootstrap algorithm for the RAxML Web servers. Syst Biol. 2008;57(5):758-71.

54. Bonnet $\mathrm{E}, \mathrm{He} \mathrm{Y}$, Billiau K, Van de Peer Y. TAPIR, a web server for the prediction of plant microRNA targets, including target mimics. Bioinformatics. 2010;26(12):1566-8.

55. Kozomara A, Griffiths-Jones S. miRBase: annotating high confidence microRNAs using deep sequencing data. Nucleic Acids Res. 2014;42(Database issue):D68-73.

56. Kinsella RJ, Kahari A, Haider S, Zamora J, Proctor G, Spudich G, et al. Ensembl BioMarts: a hub for data retrieval across taxonomic space. Database (Oxford). 2011;2011:bar030. 